\title{
Spontaneous hemothorax under rivaroxaban treatment
}

\author{
(D)Mehmet Emin Demir \\ İstanbul Yeni Yüzyıl University Private Gaziosmanpasa Hospital, Department of Nephrology, İstanbul, Turkey
}

Cite this article as: Demir ME. Spontaneous hemothorax under rivaroxaban treatment. J Health Sci Med 2021; 4(2): 246.

\section{Dear Editor,}

Rivaroxaban is a highly selective direct thrombin inhibitor indicated in stroke prevention, non-valvular atrial fibrilation, treatment of pulmonary embolism and deep vein thrombosis, prevention of recurrent venous thromboembolism and VTE after elective hip/knee replacement surgery and secondary prevention after acute coronary syndrome. Hemorrhage is the major concern of the drug use. Subdural hematoma, cerebral hemorrhage, GIS hemorrhage, and also intra-articular bleeding has been published in postmarketing reports. Here, we report a case of spontaneous hemothorax under rivaroxaban treatment.

A 73-year-old of man was consulted due to acute dyspnea and palpitation. His $\mathrm{x}$-ray pulmonary images and thorax computed tomography were compatible with hemothorax, consequently, we performed thoracentesis, and macroscopic appearance, cell count revealed hemothorax. There was no sign of any disease, such as low pleural fluid $\mathrm{pH}$ and glucose, leucocytic dominance, dysplastic cell, which would indicate an another cause of the bleeding. He was taking rivaroxaban ( $15 \mathrm{mg}$ daily) due to chronic atrial fibrillation. Hemorrhage was drained and the drug was cessated. The patient had a history of a 1-year anticoagulant treatment under warfarin therapy with very labile INR levels before rivaroxaban. We followed-up the patient with low molecular heparin therapy for one month after discharge and then switched to low dose $(2.5 \mathrm{mg}$ daily) apixaban. Hemothorax did not relapse in the three month-follow-up period (Figure 1).

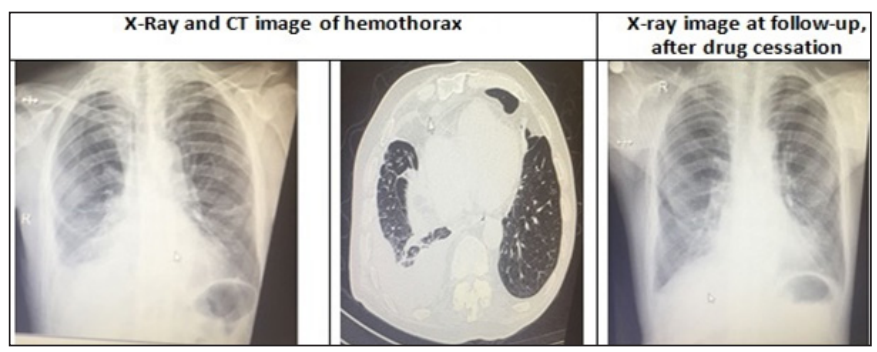

Figure. Right lung settled pleural fluid accumulation and X-ray image of the lung after recovery
Rivaroxaban (Xarelto) is a member of new anticoagulant drug class and has become favorable in the patient carrying systemic embolism risk with nonvalvular atrial fibrillation. It has predictable pharmacokinetics features, fewer food interactions, bleeding risk, and contrast to warfarin it does not need frequent INR monitoring. Despite promising features, serious hemorrhagic complications have been reported and bleeding risk is still its major challenge in clinical use. Cases of subdural hematoma, cerebral hemorrhage, GIS hemorrhage, and also intra-articular bleeding has been published (1). Spontaneous hemothorax was assumed as a rare complication until recently published case reports $(2,3)$. Here, we announce our case to keep in mind that rivaroxaban is a cause of spontaneous hemothorax, as not uncommonly.

Not: An adverse reaction notification will be sent to the related official institutions.

\section{ETHICAL DECLARATIONS}

Informed Consent: Written informed consent was obtained from all participants who participated in this study.

Referee Evaluation Process: Externally peer-reviewed.

Conflict of Interest Statement: The authors have no conflicts of interest to declare.

Financial Disclosure: The authors declared that this study has received no financial support.

Author Contributions: All of the authors declare that they have all participated in the design, execution, and analysis of the paper, and that they have approved the final version.

\section{REFERENCES}

1. Lo JC, Gerona RR. A case of rivaroxaban associated intracranial hemorrhage. West J Emerg Med 2014; 15: 375-7.

2. Lee SJ, Kim SH, Ju S. Spontaneous hemothorax as an adverse effect of Rivaroxaban Treatment. Am J Respir Crit Care Med 2016; 193: A3313.

3. Avci A, Kocer M, Gulen M. Spontaneous hemothorax secondary to oral anticoagulation therapy. JAEMCR 2014; 5: 135-7. 